\title{
MEDIDA DE EFICIENCIA TÉCNICA EN LA EDUCACIÓN MEDIA DE AMÉRICA LATINA: PRUEBAS PISA
}

Rafael Antonio Viana Barceló

Universidad Industrial de Santander

Colombia

Yehisen Frederick Urbina Fernández

Universidad Industrial de Santander

Colombia 
Panorama Económico, Vol. 27 - No. 1 (Enero - Marzo de 2019), pp. 39-59

Rafael Antonio Viana Barceló

Yehisen Frederick Urbina Fernández

JEL: I20, I21, I28

\title{
Medida de eficiencia técnica en la educación media de América Latina: Pruebas PISA
}

\section{Resumen}

En el presente estudio se evalúa la eficiencia técnica de estudiantes de 8 países de América Latina que participaron en las pruebas PISA 2015. El estudio se desarrolló bajo una metodología no paramétrica. Para ello, se empleó un modelo FDH con orientación al producto con un enfoque de estimaciones robustas y una descomposición de Metafrontera, esto último permite descomponer la eficiencia total de los estudiantes, entre la atribuible al estudiante y la atribuible al país. Los datos utilizados en el estudio provienen de las pruebas PISA 2015. Los datos utilizados corresponden 35,880 observaciones de igual número de estudiantes que presentaron la prueba mencionada. Los resultados revelan que la mayor parte de la ineficiencia se debe a los propios estudiantes. Identificando a Uruguay y Brasil como los países donde se presenta el menor puntaje de ineficiencia. Y a México y República Dominicana como los que presentan un mayor puntaje de ineficiencia.

Palabras clave: Eficiencia, fdh, metafrontera, insumo, productos.

\section{Mesure d'efficacité technique dans l'enseignement secondaire latino-américain: tests PISA}

\section{Résumé}

Cette étude évalue l'efficacité technique des étudiants de 8 pays d'Amérique latine qui ont participé aux tests PISA 2015. L'étude a été développée selon une méthodologie non paramétrique. Pour cela, un modèle FDH orienté produit a été utilisé avec une approche d'estimations robustes et une décomposition Méta-frontière. Cette dernière permet de décomposer l'efficience totale des étudiants, entre celle imputable à l'étudiant et celle imputable au pays. Les données utilisées dans l'étude proviennent des tests PISA 2015. Les données utilisées correspondent à 35880 observations du même nombre d'étudiants ayant présenté le test précité. Les résultats révèlent que la majeure partie de l'inefficacité est due aux étudiants eux-mêmes. Identifier l'Uruguay et le Brésil comme les pays ayant le score d'inefficacité le plus faible. Et au Mexique et en République Dominicaine comme ceux avec le score d'inefficacité le plus élevé.

Mots clés: Efficacité, fdh, méta frontière, intrants, produits.

\section{Technical efficiency measure in Latin American secondary education: PISA tests}

\begin{abstract}
This study evaluates the technical efficiency of students from 8 Latin American countries that participated in the PISA 2015 tests. The study was developed under a non-parametric methodology. For this, a product-oriented FDH model was used with a robust estimations approach and a Meta-frontier decomposition. The latter allows decomposing the total efficiency of the students, between that attributable to the student and that attributable to the country. The data used in the study comes from the PISA 2015 tests. The data used correspond to 35,880 observations from the same number of students who presented the aforementioned test. The results reveal that most of the inefficiency is due to the students themselves. Identifying Uruguay and Brazil as the countries with the lowest inefficiency score. And to Mexico and the Dominican Republic as those with the highest inefficiency score.
\end{abstract}

Keywords: Efficiency, fdh, metafrontier, input, products. 


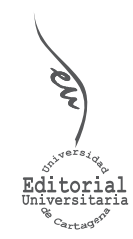

\section{Medida de eficiencia técnica en la educación media de América Latina: Pruebas PISA}

INFORMACIÓN DEL ARTíCULO

Recepción del artículo: 04/06/2018

Concepto de evaluación: $17 / 07 / 2018$

Aceptación del artículo: 25/08/2018
Rafael Antonio Viana Barceló* Universidad Industrial de Santander, Colombia

Yehisen Frederick Urbina Fernández Universidad Industrial de Santander, Colombia

\section{INTRODUCCIÓN}

El uso de pruebas estandarizadas como TIMSS ${ }^{1}$ (Trends in International Mathematics and Science Study), PIRLS ${ }^{2}$ (Progress in International Reading Literacy Study) y PISA ${ }^{3}$ (The Programme for International Student Assessment), se han hechopopulares en la medición de logros educativos porque al tiempo que facilitan el análisis de los determinantes y el impacto de las habilidades cognitivas de los estudiantes al interior de los países, permiten realizar comparaciones de los sistemas educativos entre los diferentes países donde se aplican. Dichas pruebas pueden presentar desventajas atribuibles al número limitado de observaciones por país, posibles sesgos de factores no observados de cada país (por ejemplo, la cultura), y el carácter transversal de los datos (Bray \& Thomas, 1995; Hanushek \& Woessman, 2011). De igual forma, Rowe (2000), resalta las limitaciones del uso de indicadores en los exámenes como medidas de responsabilidad en la escuela y a nivel del sistema, como medidas de los resultados del aprendizaje de los estudiantes ${ }^{4}$.

Deacuerdocon Hanushek(1979), la correcta medición del logroeducativoyla comprensión de sus determinantes, es de gran utilidad para el diseño de políticas referentes a temas como la rendición de cuentas de los maestros, los sistemas de financiamiento educativo y de integración escolar.

\footnotetext{
*Profesor Asociado de la Escuela de Economía y Administración de la Universidad Industrial de Santander (Colombia). Autor de correspondencia: ranviana@uis.edu.co

1. Evaluación internacional de conocimientos de matemáticas y ciencias de los estudiantes inscritos en los grados cuarto y octavo de todo el mundo.

2. Estudio internacional del logro de la lectura en estudiantes de cuarto grado. Está dirigido por la Asociación Internacional para la Evaluación del Logro Educativo

3. Encuesta trienal, evalúa hasta qué punto los estudiantes de 15 años cerca del final de la educación obligatoria adquirieron conocimientos y habilidades clave que son esenciales para la plena participación en las sociedades modernas.

4. Este tema no se discutirá dentro del marco de este trabajo.
} 
La literatura (Ruggiero, 1996; Täht \& Must, 2013; Zhao, y otros, 2008) empírica reconoce que una gran proporción de estudios referentes al desempeño educativo realizan un análisis inadecuado del tema, lo cual, deriva en falencias tales como no tener en cuenta los contextos económico, cultural y social de cada país.

Realizar comparaciones directas sin una metodología adecuada, deriva en sesgos y conclusiones erróneas. Por lo tanto, es evidente la necesidad de un estudio que analice la educación desde una perspectiva de eficiencia que permita y facilite realizar comparaciones entre estudiantes de los países participantes en las diferentes evaluaciones. Por ello, el objetivo de este trabajo es medir la eficiencia de los estudiantes latinoamericanos que presentaron las pruebas PISA 2015 y el valor agregado que imprime cada uno de los países en sus estudiantes.

\section{CONCEPTO DE EFICIENCIA TÉCNICA EN LA EDUCACIÓN}

Urwick y Junaidu (1991), diferencian dos orientaciones que divergen al analizar el concepto de calidad en educación, la eficiencia técnica y la orientación pedagógica. Dichos autores postulan que la eficiencia técnica se orienta sobre la base de la provisión de recursos de la escuela (especialmente profesores, materiales educativos y tiempos de enseñanza), de sus efectos sobre el logro académico y las consecuentes prioridades de inversión. La orientación pedagógica se enfoca en las habilidades de los profesores, las capacidades de organización de la escuela y aspectos curriculares como componentes primordiales de calidad.

El concepto de eficiencia técnica es mencionado por primera vez por Koopmans (1951), que lo definió como la capacidad de producir la mayor cantidad de producto con la menor cantidad de insumos o recursos 5 . Por ello, es necesario concebir a las organizaciones como un conjunto coordinado y único de recursos y capacidades heterogéneas que se generan, desarrollan y mejoran con el paso del tiempo, siendo estos lo que explica las diferencias de desempeño (Barney, 1991; Bass \& Avolio, 1997). Posteriormente, se desarrollan las primeras aplicaciones empíricas (Farrell M. , 1957; Farrell \& Fieldhouse, 1962; Schmidt, 1976).

En el contexto educativo se ha analizado el valor agregado que generan las diferentes escuelas en los alumnos (Sammons, Thomass, Motimore, Owen, \& Pennell, 1996), no obstante, los resultados de las evaluaciones a nivel de alumnos pueden diferir de los estudios a nivel de la escuela (Kramer, 1983). Inicialmente se han utilizado regresiones MCO o de variable dependiente limitada (Probit, Tobit, Logit), cuyos problemas ya han sido puestos en manifiesto (Balaguer, Prior, \& Tortosa, 2007; Banker \& Natajaran, 2008; Simar \& Wilson, 2007; Simar \& Wilson, 2011). Por ello, se ha propuesto la aplicación de modelos no paramétricos con datos a nivel de alumnos, anidados en colegios (Portela \& Thanassoulis, 2001; Thanassoulis, 1999; Thanassoulis \& Portela, 2002).

En el estudio de eficiencia técnica del sector educativo, se destaca la utilización de modelos estadísticos de multinivel (Goldstein, 2003; Johnes, 2006; Sammons, Nuttall, \& Cuttance,

5. Son cantidades de factores disponibles que posee o controla una empresa, siendo de naturaleza diversa y comprendiendo factores físicos, tecnológicos, humanos y organizativos (Cuervo, Á., 1993). 
1993), el Análisis Envolvente de Datos, DEA (Altamirano \& Peniche, 2014) y modelos Free Disposal Hull, FDH ${ }^{6}$ (Cordero, Santín, \& Simancas, 2017; De Witte \& Kortelainen, 2013; Haelermans \& De Witte, 2012).

\section{EL MODELO FREE DISPOSAL HULL (FDH)}

Entre las técnicas no paramétricas, los estudios con DEA son los más utilizados en la literatura; aun así, la principal crítica que recae sobre este es modelo es que compara los datos con unidades ficticias (o virtuales) construidas a partir de combinaciones convexas entre las unidades (Cooper, Seiford, \& Tone, 2000). Por lo contrario, dicha falencia no se presenta en la técnica FDH, que compara los datos con unidades reales tomadas del conjunto de producciones factible de la muestra (Deprins, Simar, \& Tulkens, 2006) teniendo aplicaciones en el campo educativo, por ejemplo, la medición de eficiencia del sistema público y privado (Cherchye, De Witte, Ooghe, \& Nicaise, 2010), la evaluación del rendimiento de los profesores (De Witte \& Rogge, 2011) y las diferencias en la educación a nivel de país (De Witte, Thanassoulis, Simpson, Battisti, \& M, 2010; Thieme, Prior, \& Tortosa, 2013).

No obstante, el modelo FDH es muy sensible a la existencia de valores atípicos, estos pueden provocar una medida errada de la frontera eficiente y por ende de la eficiencia de cada unidad. Para la solución de este problema, Cazáls, Florens y Simar L (2002), sugieren evaluar la eficiencia de las observaciones relativas a una frontera parcial, usando solamente $\mathrm{m}<\mathrm{n}$ observaciones y empleando extracción con reemplazo entre aquellas yi tal que xi $\leq \mathrm{x}$. Gracias a la replicación de las extracciones a las que se les denomina robustas de orden-m, es posible realizar inferencia estadística (Daraio \& Simar, 2005) La metodología de orden-m se ha utilizado para comparar grupos de distintos tamaños, dado que mediante un "m" igual para todos los grupos, se reducen los sesgos (Zhang \& Bartels, 1998; De Witte \& Marques, 2009). Para ello, De Witte y Marques (2009), recomiendan utilizar "m" igual al mínimo de observaciones por unidad.

Es posible que algunas de las observaciones puedan estar situadas más allá de la frontera de dicha muestra, es decir, que no están contenidas en ese subconjunto. De aquí se pueden derivar dos situaciones: i) las observaciones pueden ser súper eficientes (Andersen \& Petersen, 1993), lo que se traduce como que dicha unidad obtiene rendimientos superiores a los que se podría esperar dados unos niveles de insumo; ii) la inviabilidad podría ocurrir, en medida que no se cuenta con niveles de referencia adecuados dentro de la muestra para estimar la salida máxima factible para una observación que no esté dentro de la muestra. La solución a este problema es calcular la eficiencia orientada al insumo.

\section{EL ENFOQUE DE METAFRONTERA}

En la mayoría de las aplicaciones los datos se encuentran contenidos dentro de unidades y estos contenidos a su vez dentro de entidades. A esto en la literatura se le conoce como datos jerárquicos o multinivel. El enfoque metafrontera mide la eficiencia de las unidades

6. Free Disposal Hull (FDH), es un modelo de medida de la eficiencia técnica en la utilización de recursos disponibles, para lo cual se basa en observaciones reales para la creación de una frontera de mejores prácticas. 
evaluadas en relación a las fronteras parciales de las mejoras prácticas, con especial aplicación en datos jerárquicos (Battese \& Rao, 2002; Battese, Rao, \& O’Donnell, 2004) debido que al no tener en cuenta estos aspectos podría conllevar a sesgos en los resultados.

El enfoque de Metafrontera en el campo educativo permite descomponer el logro del alumno evaluado en una parte atribuible al alumno y una parte atribuible al sistema educativo. Es decir, una descomposición de frontera a nivel de estudiante y sistema educativo (Ruggiero, 2000; Thanassoulis \& Portela, 2002).

Racine, Hart y Li (2006), proponen una solución para la medida de la significancia en modelos de regresión no paramétricos, aplicando pruebas bootstrap como equivalente a t-student para esto Daraio y Simar (2005, p 103) recomiendan hacer un mínimo de 200 replicaciones.

\section{ANÁLISIS TÉCNICO DE LA EDUCACIÓN}

Referente a medidas de eficiencia en el campo educativo son muchos los trabajos que se han desarrollado, en los que se analizan aspectos como diferencias en escuelas públicas y privadas (Dronkers \& Robert, 2008; Vandenberghe \& Robin, 2004), los efectos del tracking ${ }^{7}$ (Brunello \& Checchi, 2007; Schuetz \& HL, 2008), los antecedentes culturales (Coco \& Lagravinese, 2014), valores contextuales (Coelli, Rao, O’Donnell, \& Battese, 2005), el capital humano en las escuelas (Bradley, Johnes, \& Millington, 2001; Worthington, 2001), número de profesores por cantidad de estudiantes (Brennan, Haelermans, \& Ruggiero, 2014; Cordero, Pedraja, \& Santín, 2010; Essid, Ouellette, \& Vigeant, 2014), tiempo de instrucción a los estudiantes (Lavy, 2015; Rivkin \& JC, 2015), recursos físicos como número de computadores por estudiante (Spiezia, 2010), datos socioeconómicos de los estudiantes (Agasisti, 2014; Crespo, Pedraja, \& Santín, 2014; Thieme, Prior, \& Tortosa, 2013) y aspectos de la personalidad como la escrupulosidad en el rendimiento académico de los estudiantes (Borghans \& Schils, 2012; Heckman, 2011).

Si bien, estos estudios demuestran una relación de dichas variables con los logros de los estudiantes, estudios como el de Cordero et al (2017) muestra que los recursos físicos de las instituciones tienen poco efecto en dichos logros. Hanushek y Woessman (2011) encuentran que si bien, las medidas de los insumos cuantitativos muestran poco impacto, estructuras institucionales y de la calidad de la fuerza docente, pueden proporcionar grandes diferencias internacionales en el nivel y la equidad de los logros estudiantiles.

Las pruebas PISA han servido de recurso para múltiples estudios donde se analizan los resultados a nivel de país (Afonso \& Aubyn, 2006; Aristovnik \& Obadic, 2014; Giambona, Vassallo, \& Vassiliadis, 2011; Thieme, Giménez, \& Prior, 2012). También, se han realizado estudios en los que se aplican micro fronteras al analizar la eficiencia con datos de PISA (De Jorge \& Santín, 2010). La aplicación del modelo FDH ha tomado popularidad en el contexto educativo. Algunos autores han incluido en sus propuestas, aplicaciones tales como ampliar el modelo robusto FDH para incorporar variables exógenas (Daraio \& Simar, 2007). Otra propuesta interesante es la de Leleu (2006) para una programación lineal del modelo FDH.

7. Es un proceso de seguimiento realizado en algunas escuelas en las que se separa a los alumnos en grupos según su capacidad académica para todas o algunas clases. 
Con el fin de contrastar la fiabilidad del modelo FDH, De Witte. et al (2010), realizan una comparación del modelo paramétrico de multinivel y modelo FDH con un enfoque de metafrontera, para calcular la eficiencia técnica en 3017 niñas de 21 escuelas de único género en Gran Bretaña. Los resultados demostraron que los dos métodos coinciden en la variación en el logro del alumno, en la proporción atribuible al alumno y a la escuela. Por ello, se considera el FDH como un procedimiento natural para establecer objetivos, ya que es fácil fijar metas individuales para los alumnos en el marco del mismo.

De igual forma, Thieme, Prior y Tortosa-Ausina, (2011), aplican una metodología FDH robusta de orden-m, en una muestra de 277 centros de educación básica de Chile ( colegios privados, privados subvencionados (concertados) y municipales (públicos); y de los que se cuenta con información completa acerca de recursos, capacidades organizativas y resultados a través de la prueba estandarizada de rendimiento, denominada pruebas $\operatorname{SIMCE}^{8}$ (Sistema de Medición de la Calidad de la Educación). Dicha prueba, se implementan de manera censal para alumnos de cuarto y octavo grado en las áreas de matemáticas, lenguaje y ciencias. Los resultados de eficiencia técnica son coherentes con los encontrados en estudios previos para Chile, que señalan que la ineficiencia técnica de gestión promedio bordea el 6\%. La eficiencia técnica que muestran los colegios privados pagados es superior a los privados subvencionados y, a su vez, la de estos es superior a la de los colegios públicos municipales. Sin embargo, las diferencias entre los dos tipos de colegios privados no son estadísticamente significativas. La brecha disminuye considerablemente al controlar por nivel socioeconómico de las escuelas, aunque persiste un mejor desempeño de los privados pagados, seguido por los privados subvencionados y finalmente los municipales.

Respecto a estudios diferenciales en eficiencia a nivel de país cabe resaltar el realizado por Cordero, et al. (2017), quienes realizaron un estudio comparativo entre 16 países de la Unión Europea, mediante un modelo robusto de orden-m con un enfoque de metafrontera en una muestra de 2.398 escuelas obtenida de las pruebas PIRLS 2011. En su estudio, los autores tomaron como producto los resultados en lectura y como insumos las variables de información de antecedentes sobre el estatus socioeconómico de los estudiantes, el ambiente escolar y las prácticas de instrucción. Además, utiliza variables económicas como el ingreso per-cápita e indicadores de valores culturales tales como trabajo duro, responsabilidad y perseverancia (datos obtenidos de la base WVS por sus siglas en inglés World Values Survey ${ }^{9}$ ), para diferenciar a nivel de país. Los autores señalan que el ranking difiere cuando se introducen este tipo de variables debido a las diferencias en los recursos disponibles de cada país. Los resultados indican que la transmisión intergeneracional de habilidades no cognitivas, tales como responsabilidad o perseverancia están significativamente relacionados con la eficiencia escolar, mientras que la mayoría de los factores escolares no parecen tener una influencia significativa en el rendimiento escolar.

8. El SIMCE hace parte del sistema aseguramiento de la calidad de la educación escolar (Chile), es una institución de sentido público tiene como funciones centrales evaluar y orientar al sistema educativo para contribuir al mejoramiento de la calidad de las oportunidades educativas.

9. Es una red global de científicos sociales que estudia el cambio de valores y su impacto en la vida social y política. La encuesta, iniciada en 1981, busca utilizar los grupos de investigación más rigurosos y de alta calidad en cada país. La WVS consiste en encuestas representativas a nivel nacional realizadas en casi 100 países que contienen casi el 90 por ciento de la población mundial, utilizando un cuestionario común. La WVS es la más grande investigación no comercial, transnacional, de series temporales de creencias y valores humanos. 


\section{METODOLOGÍA}

En el presente estudio, se procedió a medir la eficiencia de cada uno de los estudiantes a nivel de país, posteriormente se midió la eficiencia total, que es la eficiencia del estudiante al compararse con alumnos de todos los países. Esto permite descomponer la ineficiencia y determinar que parte es atribuible al país.

Para estimar las diferentes medidas de eficiencia, se utilizó la técnica FDH con orientación al producto y una descomposición de Metafrontera. El modelo FDH, propuesto inicialmente por Deprins D et al. (1984) desarrolla la siguiente metodología: considerando un conjunto de datos $(\mathrm{x}, \mathrm{y})$ insumos y productos respectivamente, que pertenecen a un conjunto factible de producción $Y$, para los cuales se desea calcular la eficiencia, primero es necesario estimar la frontera eficiente de producción (desconocida) y a partir de esto se calcula la distancia entre cada punto observado y la frontera eficiente de producción. En el gráfico 1, la eficiencia del punto A, viene dado por la distancia entre este y la frontera parcial.

Ahora bien, suponga una lista de insumos (q) y productos (p), tal que se cumpla:

$$
\mathbb{Y}=\left\{(x, y) \mid x \in \mathbb{R}_{+}^{q}, y \in \mathbb{R}_{+}^{p},(x, y) \text { es factible }\right\}
$$

Así el FDH orientado al producto, se obtiene resolviendo la siguiente ecuación:

$$
F D H(x, y)=\operatorname{Max}\left\{\alpha \mid \alpha y \leq \sum_{i=1}^{N} \gamma_{i} y_{i} ; x \geq \sum_{i=1}^{N} \gamma_{i} x_{i} ; \sum_{i=1}^{N} \gamma_{i}=1 ; \gamma_{i} \in[0,1] ; i=1, \ldots n\right\}
$$

Para calcular la frontera de producción se realizan dos supuestos: i) libre disponibilidad de insumos y de productos. Si se trata de un punto eficiente este efecto será igual a 1. Si el efecto es mayor a 1, este punto será ineficiente. Por otro lado, puede ocurrir que un individuo obtenga resultados superiores de los que se espera a partir de sus recursos disponibles, en este caso el efecto será menor a 1 y significara un punto súper- eficiente.

Como ya se había mencionado, el modelo FDH es muy sensible a los valores atípicos, Cazals et al (2002), proponen una estimación robusta de orden-m, sugiriendo evaluar la eficiencia de las observaciones relativas a una frontera parcial, usando solamente $\mathrm{m}<\mathrm{n}$ observaciones, mediante extracción con remplazo entre aquellas yi tal que $\mathrm{xi} \leq \mathrm{x}$.

Elenfoque de Metafrontera, surge debido a quela mayoría de los datos tienen una estructura multinivel. Este enfoque permite medir la eficiencia de las unidades evaluadas en relación con las fronteras separadas de mejores prácticas. También, permite observar la eficiencia de los estudiantes a nivel país (frontera individual) y a nivel de conjunto en una frontera total (metafrontera) conformada por las observaciones de todos los países evaluados. En el gráfico 1, el punto A representa cada estudiante del que se tiene información en las pruebas PISA 2015, la frontera parcial hace relación a las mejores prácticas de cada país y la Metafrontera representa las mejores prácticas del conjunto formado por todos los países incluidos en el estudio. De esta manera, el procedimiento para evaluar la eficiencia de cada país, es medir la distancia entre la frontera de cada país respecto a la Metafrontera. Así la eficiencia general se puede descomponer como (Thanassoulis \& Portela, 2002): 
(BD/BA): eficiencia general.

(BD/BC): efecto país.

(BC/BA): efecto estudiante.

$(\mathrm{BD} / \mathrm{BA})=(\mathrm{BD} / \mathrm{BC}) *(\mathrm{BC} / \mathrm{BA})$.

\section{Ilustración. El enfoque de Metafrontera}

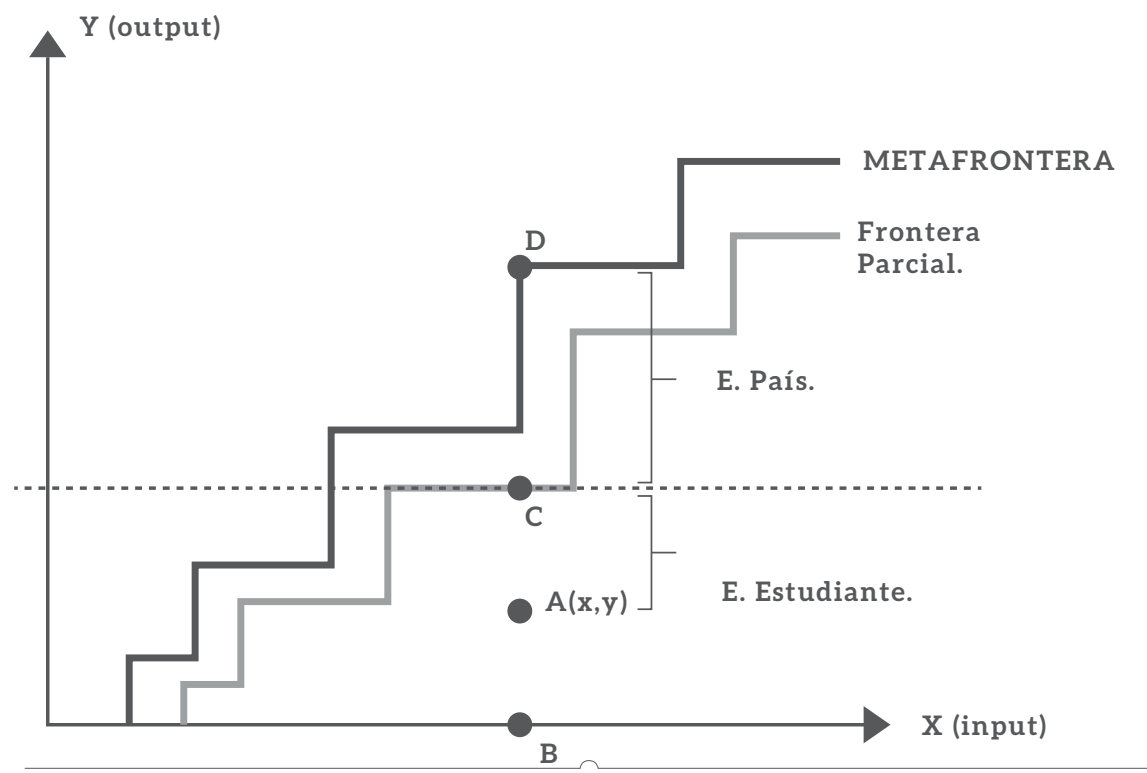

\section{DATOS}

Los datos para este estudio provienen de las pruebas PISA 2015. Estos datos proporcionan una comparación internacional sobre los rendimientos en lectura, matemáticas y ciencias de los estudiantes de décimo grado (15 años), así como una amplia base de información acerca de los estudiantes, centros educativos y planta de profesorado.

La muestra inicial estuvo conformada por 82.202 observaciones, dentro de las cuales se tenían datos de Argentina y Puerto Rico. No obstante, debido a datos faltante para estos países no pudieron ser incluidos en al análisis. Tras la selección de variables y la depuración de la base de datos, la muestra final quedó conformada por 35,880 estudiantes, pertenecientes a 8 países de América Latina descritos en la tabla 1. El país con mayor representación en la muestra fue Brasil (18.79\%), seguido por Colombia (16.66\%) y la menor participación fue República dominicana (6.28\%). Esta distribución en la muestra se encuentra correlacionada con el número de estudiantes de 15 años en cada país, ya que la metodología PISA extrae una muestra que sea representativa de la población evaluada. 
Tabla 1.

Distribución de la muestra.

\begin{tabular}{lcc}
\hline País & Estudiantes & Porcentaje \\
\hline Brasil & 5.546 & 15,46 \\
Chile & 4.426 & 12,34 \\
Colombia & 6.452 & 17,98 \\
Costa Rica & 3.903 & 10,88 \\
R. Dominicana & 2.114 & 5,89 \\
México & 5.277 & 14,71 \\
Perú & 5.090 & 14,19 \\
Uruguay & 3.072 & 8,56 \\
& & 100,00
\end{tabular}

Fuente: Elaboración propia a partir de los datos disponibles en www.oecd.org/pisa/pisaenespaol.htm.

En la tabla 2, se muestra las puntuaciones medias de cada país en cada área evaluada, además se encuentran ordenadas según el ranking presentado por PISA para las pruebas 2015. Es necesario recordar que la metodología de PISA controla todos los posibles sesgos que pudieran cometerse, es decir, la técnica de muestreo tiene en cuenta estudiantes inmigrantes, estratos, entre otros. De este modo, la muestra es totalmente representativa de la población.

Tabla 2.

Puntuaciones medias en ciencias, lectura y matemáticas.

\begin{tabular}{l|c|cc}
\hline País & P. Media en ciencias & P. Media en lectura & P. Media en matemáticas \\
\hline Chile & 447 & 459 & 423 \\
Uruguay & 435 & 437 & 418 \\
Costa Rica & 420 & 427 & 400 \\
Colombia & 416 & 425 & 390 \\
México & 416 & 423 & 408 \\
Brasil & 401 & 407 & 377 \\
Perú & 397 & 398 & 387 \\
R. Dominicana & 332 & 358 & 328 \\
& & & \\
\hline
\end{tabular}

Fuente: OCDE, PISA 2015, database. http://dx.doi.org/10.1787/888933431961. 


\section{VARIABLES}

\section{Productos}

Como productos se seleccionaron los resultados individuales en lectura, matemáticas y ciencia. Dichos resultados se presentan en los denominados valores plausibles. Estos valores se extraen aleatoriamente de la distribución de resultados de los alumnos, construida a partir de las respuestas que dan a las preguntas que se le plantean en el test de conocimientos. A partir de esas respuestas y utilizando la teoría de la respuesta al ítem de Rasch (1980) se estima la distribución de resultados de los alumnos y de dicha distribución se extraen los valores plausibles. La idea que subyace en esta forma de presentar los resultados es que resulta imposible conocer con exactitud cuáles son los conocimientos de los alumnos en tan poco tiempo de evaluación. En este trabajo se utilizó el primer valor plausible de cada área evaluada como producto.

\section{Insumos}

Para la selección de los insumos (tabla 3), en la literatura se identifica que la mayoría de los documentos empíricos que intentan medir eficiencia en la educación por lo general incluyen medidas de recurso humano y de capital (De Witte \& López, 2015). En el presente estudio, se implementó análisis de componentes principales ${ }^{10}$ con el objetivo de tener una única medida de los datos socioeconómicos, recursos físicos etc... que se tuvieron en cuenta por sus características determinísticas de mejores logros académicos. Dichos determinantes se encuentran descritos en la tabla 3.

Tabla 3.

\section{Descripción de componentes empleados.}

\begin{tabular}{l|l} 
C1 & $\begin{array}{l}\text { Elementos de estudio que dispone el estudiante en su hogar (libros, } \\
\text { computador, internet etc..). }\end{array}$ \\
C3 & $\begin{array}{l}\text { Indicador de datos socio económicos del estudiante (elementos de riqueza, } \\
\text { numero de autos, celulares, baños, computadores etc..). }\end{array}$ \\
C4 & $\begin{array}{l}\text { Ausentismo escolar (reportado por el estudiante y por el director } \\
\text { de la institución). } \\
\text { Ambiente en el aula de clase (hay ruido y desorden, los estudiantes } \\
\text { no escuchan etc..). } \\
\text { Dotación de tecnología de la institución (computadores, internet, tableros } \\
\text { interactivos etc...) } \\
\text { C6 }\end{array}$
\end{tabular}

10. Posterior al Análisis de componentes principales, se procedió a re-escalar los valores. Esto debido a que la naturaleza del FDH no permite la existencia de valores negativos o ceros. 
Tabla 3.

Descripción de componentes empleados.

\begin{tabular}{l|l}
\hline C7 & $\begin{array}{l}\text { Autonomía de la institución. } \\
\text { Cualificación de la planta profesoral (profesores con maestría, } \\
\text { doctorado etc...) }\end{array}$ \\
C9 & $\begin{array}{l}\text { Número de horas impartidas a la semana en clase de lectura. } \\
\text { C10 }\end{array}$ \\
C11 & $\begin{array}{l}\text { Número de horas impartidas a la semana en clase de matemáticas. } \\
\text { C12 }\end{array}$ \\
& $\begin{array}{l}\text { Tipo de localidad donde se encuentra ubicada la institución (villa, } \\
\text { pueblo, ciudad etc...) }\end{array}$
\end{tabular}

Tabla 4.

Descriptivos de los insumos por país.

\begin{tabular}{llllll|l|l|lc}
\hline & Uruguay & Brasil & Perú & Colombia & $\begin{array}{c}\text { Costa } \\
\text { Rica }\end{array}$ & Chile & México & $\begin{array}{c}\text { República } \\
\text { Dominicana }\end{array}$ \\
\hline C1 & 3,30 & 3,76 & 3,40 & 3,63 & 4,08 & 3,27 & 4,23 & 3,51 \\
C2 & 2,66 & 2,85 & 3,74 & 3,10 & 2,89 & 2,82 & 3,33 & 3,22 \\
C3 & 5,66 & 4,77 & 4,19 & 4,90 & 5,02 & 6,35 & 4,80 & 4,60 \\
C4 & 5,79 & 5,58 & 6,19 & 5,95 & 6,21 & 5,83 & 6,02 & 5,99 \\
C5 & 2,54 & 2,27 & 2,37 & 2,28 & 2,51 & 2,19 & 2,12 & 2,24 \\
C6 & 1,00 & 1,00 & 1,27 & 1,57 & 1,00 & 1,21 & 1,61 & 2,28 \\
C7 & 3,55 & 2,62 & 2,51 & 3,12 & 2,57 & 3,26 & 2,64 & 3,07 \\
C8 & 1,50 & 2,08 & 2,53 & 2,18 & 1,49 & 3,35 & 1,63 & 2,21 \\
C9 & 3,64 & 4,15 & 5,68 & 3,68 & 4,72 & 6,39 & 4,49 & 4,25 \\
C10 & 4,02 & 4,22 & 6,36 & 3,98 & 5,07 & 6,80 & 4,60 & 4,28 \\
C11 & 5,14 & 3,85 & 4,76 & 3,79 & 5,83 & 6,15 & 4,68 & 4,14 \\
C12 & 3,47 & 4,68 & 4,16 & 5,78 & 4,15 & 5,14 & 4,04 & 5,26
\end{tabular}

\section{RESULTADOS}

La estimación de la eficiencia general se obtuvo utilizando toda la muestra final a través de la metodología de orden-m con una orientación al producto. Por otro lado, para hallar el efecto estudiante y el efecto país se calcularon 8 fronteras parciales, una por cada país. Para calcular la frontera general se utilizó un $\mathrm{m}=50$ y 400 replicaciones y para las fronteras parciales un $\mathrm{m}=50$ y 200 replicaciones.

En la tabla 5, se muestran los puntajes promedio de eficiencia estimados para cada país. Al analizar la eficiencia total, es decir, cuando los estudiantes son comparados con sus iguales de todos los países, se destaca que en promedio los alumnos en todos los países se muestran ineficientes. No obstante, también se evidencia que existen alumnos con valores de eficiencia inferiores a 1, es decir, súper-eficientes. En Costa 
Rica, se registra la observación más súper-eficiente (0,794).

En promedio, Uruguay $(1,048)$ y Brasil $(1,0508)$ son los países donde el nivel de ineficiencia es menor; estos valores de eficiencia indican que, si todos los estudiantes hubiesen mostrado comportamientos eficientes, los resultados podrían mejorar $4,8 \%$ y $5,08 \%$ respectivamente. En contra parte, los países donde se registra un mayor nivel de ineficiencia son México (1,0935) y Republica dominicana (1,0959). El puntaje promedio de la eficiencia total fue 1,0787. Dicho puntaje señala que si todos los estudiantes se hubiesen desempeñado tan eficientemente como los ubicados sobre la frontera de mejores prácticas los puntajes de las pruebas podrían aumentar en promedio en un $7,87 \%$.

Se puede apreciar que Chile a pesar de estar ubicado en los últimos puestos del ranking, es el país donde se presentó mayor porcentaje de estudiantes súpereficientes (25,64\%), no obstante, en este mismo país fue donde se evidencio el mayor número de estudiantes ineficientes (56,17\%), evidenciando una enorme desigualdad entre los estudiantes de este país. Por la misma línea, República Dominicana $(56,24 \%)$ y Brasil $(54,72 \%)$ son los países donde se observa un mayor porcentaje estudiantes eficientes.

El efecto país explica la proporción de la ineficiencia que es atribuible al país donde se desenvuelve el estudiante; los países donde este efecto es menor son Uruguay $(1,0145)$ y Brasil $(1,0152)$. Por su parte, el efecto estudiante explica la proporción de la ineficiencia que se le atribuye al propio estudiante; los países donde este efecto es menor son República Dominicana $(1,0238)$ y Costa Rica $(1,0356)$.

Al descomponer la eficiencia, se puede apreciar que en promedio una mayor fracción de la ineficiencia total se debe a los propios estudiantes (1,0442). Sin embargo, lo anterior difiere entre países, por ejemplo, gran parte de la ineficiencia que opera en R. Dominicana $(1,0751)$ y Costa Rica $(1,0578)$ corresponde al efecto país, mientras que este mismo efecto en Brasil y Uruguay es apenas significativo.

Este hallazgo puede tener un efecto relevante al compararlo con los obtenidos por Cordero, et al. (2017), en este trabajo realizado para paises de la union europea, se identificó una menor relevancia del efecto del pais sobre el logro academico que la concerniente a las propias escuelas. En este sentido, se logra identificar una diferencia entre los dos grupos de paises, latino americanos y europeos. Lo cual puede deberse a que los sistemas de enseñanza en los países latinos americanos posean caracteristicas similares, no indicando esto que sean iguales.

Al comparar el porcentaje de estudiante súper-eficientes ubicados en la frontera parcial, es decir, cuando se realiza la comparativa entre estudiantes de mismo país (efecto estudiante) y los de la frontera general (eficiencia total), se tiene que el porcentaje de estudiantes súper-eficientes disminuye de un 15,28\% a un 4,28\%, esto se debe a que los estudiantes dentro de la frontera parcial se encuentran en condiciones mucho más homogéneas y por ende el número de estudiantes que logran destacar súper-eficientemente es más reducido. Por otro lado, el porcentaje de estudiantes eficientes aumenta de 41,36\% a 71,10\%, induciendo a una reducción en el porcentaje de estudiantes que incurren en conductas ineficientes (de 43,37\% a $24,62 \%)$. 
Es importante prestar atención en los casos de R. Dominicana y Colombia. El primero, cuando se compara solo con estudiantes de su mismo país (efecto estudiante), resulta ser el país donde se incurre en menor ineficiencia. Pero al compararse con estudiantes de todos los países de la muestra (efecto total), presenta los mayores niveles de ineficiencia. Esto se explica debido a que un estudiante puede ser eficiente al comparase con sus pares del mismo país, pero al ser comparado con estudiantes de otros países se evidencia que podría tener mayores logros con base en los recursos de los que dispone, es decir, en los otros países existen estudiantes que con la misma cantidad de recursos obtienen mayores puntuaciones en las pruebas de evaluación. En este caso, la diferencia entre un estudiante y el otro recae en el valor agregado generado por cada país.

En el caso de Colombia ocurre lo contrario, al realizarse una comparación entre estudiantes del mismo país los niveles de ineficiencia resultan ser mayores que a los demás, esto puede ser explicado por altos niveles de desigualdad de condiciones entre los estudiantes de este país, y en gran parte por la existencia de estudiantes resilientes, que son aquellos que a pesar de disponer de muy bajos recursos logran obtener altas puntuaciones en las pruebas. Por otro lado, sus resultados globales mejoran significativamente al compararse con estudiantes de los otros países.

Tabla 5.

Descomposición de la eficiencia total.

\begin{tabular}{|c|c|c|c|c|c|c|c|}
\hline \multirow{2}{*}{ País } & \multicolumn{7}{|c|}{ Eficiencia total } \\
\hline & Mean & Std. Dev. & Min & $\operatorname{Max}$ & $\begin{array}{l}\text { \% Súper } \\
\text { eficiente }\end{array}$ & $\%$ Eficiente & $\%$ Ineficiente \\
\hline Uruguay & 1,0480 & 0,1121 & 0,8129 & 1,7323 & $18,85 \%$ & $48,86 \%$ & $32,29 \%$ \\
\hline Brasil & 1,0508 & 0,1244 & 0,8087 & 2,2044 & $15,65 \%$ & $54,72 \%$ & $29,62 \%$ \\
\hline Perú & 1,0768 & 0,1354 & 0,8570 & 2,2095 & $12,12 \%$ & $45,60 \%$ & $42,28 \%$ \\
\hline Colombia & 1,0873 & 0,1451 & 0,8527 & 2,4062 & $14,99 \%$ & $37,62 \%$ & $47,40 \%$ \\
\hline Costa Rica & 1,0887 & 0,1410 & 0,7949 & 2,0156 & $14,17 \%$ & $37,77 \%$ & $48,07 \%$ \\
\hline Chile & 1,0897 & 0,1491 & 0,7709 & 2,1458 & $25,64 \%$ & $18,19 \%$ & $56,17 \%$ \\
\hline México & 1,0935 & 0,1434 & 0,8319 & 2,4425 & $15,79 \%$ & $31,86 \%$ & $52,36 \%$ \\
\hline $\begin{array}{l}\text { República } \\
\text { Dominicana }\end{array}$ & 1,0959 & 0,1682 & 0,9296 & 2,1204 & $5,01 \%$ & $56,24 \%$ & $38,74 \%$ \\
\hline TOTAL & 1,0787 & 0,1405 & 0,7709 & 2,4425 & $15,28 \%$ & $41,36 \%$ & $43,37 \%$ \\
\hline
\end{tabular}


Tabla 5.

Descomposición de la eficiencia total.

\begin{tabular}{|c|c|c|c|c|c|c|c|}
\hline \multirow{2}{*}{ País } & \multicolumn{7}{|c|}{ Efecto estudiante } \\
\hline & Mean & Std. Dev. & Min & Max & $\begin{array}{l}\text { \% Súper } \\
\text { eficiente }\end{array}$ & \% Eficiente & $\%$ Ineficiente \\
\hline $\begin{array}{l}\text { República } \\
\text { Dominicana }\end{array}$ & 1,0238 & 0,0766 & 0,9348 & 1,6981 & $4,82 \%$ & $79,23 \%$ & $15,94 \%$ \\
\hline Costa Rica & 1,0356 & 0,0899 & 0,8955 & 1,8718 & $3,51 \%$ & $73,33 \%$ & $23,16 \%$ \\
\hline Chile & 1,0370 & 0,0953 & 0,9562 & 2,0202 & $3,23 \%$ & $74,08 \%$ & $22,68 \%$ \\
\hline Uruguay & 1,0408 & 0,1011 & 0,9102 & 1,7559 & $4,30 \%$ & $70,83 \%$ & $24,87 \%$ \\
\hline Brasil & 1,0444 & 0,1146 & 0,8328 & 2,1996 & $5,82 \%$ & $69,19 \%$ & $24,99 \%$ \\
\hline Perú & 1,0472 & 0,1076 & 0,9300 & 1,8710 & $4,62 \%$ & $68,00 \%$ & $27,39 \%$ \\
\hline México & 1,0484 & 0,1084 & 0,9214 & 1,9197 & $4,59 \%$ & $67,06 \%$ & $28,35 \%$ \\
\hline Colombia & 1,0566 & 0,1226 & 0,9198 & 2,2920 & $3,38 \%$ & $67,06 \%$ & $29,56 \%$ \\
\hline TOTAL & 1,0442 & 0,1067 & 0,8328 & 2,2920 & $4,28 \%$ & $71,10 \%$ & $24,62 \%$ \\
\hline
\end{tabular}

Tabla 5.

Descomposición de la eficiencia total.

\begin{tabular}{|c|c|c|c|c|c|c|c|}
\hline \multirow{2}{*}{ País } & \multicolumn{7}{|c|}{ Efecto País } \\
\hline & Mean & Std. Dev. & Min & $\operatorname{Max}$ & $\begin{array}{l}\text { \% Súper } \\
\text { eficiente }\end{array}$ & $\%$ Eficiente & $\%$ Ineficiente \\
\hline Uruguay & 1,0145 & 0,1357 & 0,5658 & 1,7323 & $35,35 \%$ & $34,51 \%$ & $30,14 \%$ \\
\hline Brasil & 1,0152 & 0,1472 & 0,4546 & 2,2245 & $32,65 \%$ & $37,79 \%$ & $29,55 \%$ \\
\hline Perú & 1,0369 & 0,1557 & 0,5394 & 2,1386 & $29,55 \%$ & $31,43 \%$ & $39,02 \%$ \\
\hline Colombia & 1,0398 & 0,1680 & 0,4188 & 2,4869 & $32,87 \%$ & $25,03 \%$ & $42,10 \%$ \\
\hline México & 1,0520 & 0,1638 & 0,5279 & 2,4425 & $30,70 \%$ & $21,77 \%$ & $47,53 \%$ \\
\hline Chile & 1,0577 & 0,1629 & 0,5210 & 1,9211 & $35,95 \%$ & $13,42 \%$ & $50,63 \%$ \\
\hline Costa Rica & 1,0578 & 0,1570 & 0,5342 & 2,0156 & $27,59 \%$ & $27,77 \%$ & $44,63 \%$ \\
\hline $\begin{array}{l}\text { República } \\
\text { Dominicana }\end{array}$ & 1,0751 & 0,1775 & 0,6118 & 2,1204 & $16,27 \%$ & $44,32 \%$ & $39,40 \%$ \\
\hline TOTAL & 1,0415 & 0,1597 & 0,4188 & 2,4869 & $30,12 \%$ & $29,51 \%$ & $40,38 \%$ \\
\hline
\end{tabular}




\section{DISCUSIÓN}

Es importante destacar que los resultados aquí expuestos nos indican que se debe tener cuidado al realizar conclusiones en cuanto a que país es mejor o peor en educación basados en rankings de resultados puros de pruebas estandarizadas, pues también, se debe tener en cuenta la cantidad de recursos que se están utilizando, en qué contextos están trabajando y en qué ámbito cultural se están desenvolviendo. Por ejemplo, Chile en el ranking de resultados puros, se ubica en la primera posición (tabla 2), pero en el ranking realizado con base en las medidas de eficiencia general o total (tabla 5), obtiene una posición muy por debajo de la mayoría de los países aquí evaluados. La misma situación ocurre con Costa Rica y México. Para esto último, (Dufrechou, 2016) obtiene resultados similares mediante un análisis de eficiencia en el gasto educativo en 35 países, incluidos 11 de américa latina. Entre sus resultados indica que Costa Rica y Uruguay han ocupado generalmente posiciones medias a bajas en la puntuación de eficiencia.

La explicación a este fenómeno radica en la inadecuada utilización de los recursos en dichos países, es decir, se esperaría que con los recursos de los que estos disponen obtuvieran rendimientos superiores a los que actualmente están obteniendo; estos resultados ineficientes corresponden en mayor medida al efecto país.

Por otro lado, existen diferencias entre este y otros estudios (Deutsch, Dumas, \& Silber, 2013) donde se identifica a Brasil y Chile como los países más eficientes, mientras que Uruguay es el menos eficiente. No obstante, dichas diferencias pueden deberse a múltiples causas, por ejemplo, que son datos tomados en diferentes periodos de tiempo y la eficiencia puede variar con el tiempo y a los tipos de insumos y productos usados para el análisis en cada estudio.

\section{CONCLUSIONES}

En este estudio se midió la eficiencia de estudiantes de 8 países de América Latina que presentaron las pruebas PISA 2015, mediante un modelo de estimaciones robustas FDH donde se tuvieron en cuenta factores directamente relacionados con el estudiante y el centro educativo al que asiste. Además, se utilizó un enfoque de Metafrontera para descomponer la ineficiencia en el efecto atribuible al estudiante y el efecto país.

En el caso particular de Colombia, los resultados obtenidos indican que la mayor parte de la ineficiencia radia en aspectos propios de los estudiantes. Es necesario identificar los factores que incurren en la ineficiencia de los alumnos colombianos e implementar mecanismos que contribuyan a la reducción de dicha ineficiencia para así lograr mayores logros educativos.

En cuanto a Brasil y Perú, los cuales en el ranking PISA se ubicaban en las últimas posiciones (tabla 2) y en el ranking de eficiencia total (tabla 5) se ubicaron en las primeras posiciones. Estos dos países aun, a pesar de estar incurriendo en conductas ineficientes, logran aprovechar sus recursos de una mejor manera; dichos resultados positivos se ven mayormente impulsados por el efecto país, esto debido a que la mayor fracción de la ineficiencia es debida al propio estudiante. El caso de R. Dominicana resulta ser interesante, dado que en ambos rankings este se ubicó en la última posición. 
Por su parte, Uruguay obtuvo los mejores resultados en eficiencia total y mejora su posición respecto a los demás en el ranking de resultados puros de PISA, es decir, los resultados reflejados por Uruguay en el ranking es producto de un mejor uso de los recursos disponibles.

Es importante recordar que en este estudio se realizó una comparativa solo entre países latinoamericanos, debido a su relativa homogeneidad en cuanto factores sociales, económicos y regionales. Esto con el objetivo de no realizar comparaciones probablemente sesgadas con países que puedan presentar condiciones más favorables para mayores logros educativos.

En futuras investigaciones, valdría la pena ahondar sobre las circunstancias que llevan a que los estudiantes de R. Dominicana y Costa Rica son más eficientes y ¿Por qué los estudiantes colombianos resultan ser los más ineficientes? ¿A qué responde el mayor valor agregado de países como Uruguay y Brasil?

Del mismo modo, debe tenerse en cuenta que los datos utilizados son de carácter transversal, esto debido que PISA es una prueba que se realiza cada tres años a estudiantes de décimo grado y por lo tanto cada vez son distintos los estudiantes a los que se le aplica la prueba, lo que hace imposible hacer un estudio de carácter de panel, que sería lo óptimo. Otro aspecto importante, es que los resultados están basados en un test de competencias y existen otras cuestiones no cognitivas que deben fomentarse en las escuelas, que no se pueden medir y que son cuestiones que deben tenerse en cuenta, dado que un país puede no tener los mejores resultados en cuanto a la medición de capacidades, pero logra formar bien en valores a sus estudiantes.

\section{REFERENCIAS BIBLIOGRÁFICAS}

Afonso, A., y Aubyn, M. (2006). Cross-country efficiency of secondary education provision: A semiparametric analysis with nondiscretionary inputs. Economics Modelling 23(3), 476-491.

Agasisti, A. (2014). The efficiency of public spending on education: An empirical comparison of EU countries. European Journal of Education 49(4), 543-557.

Altamirano, A., y Peniche, R. (2014). Metodología AED-RNA para la estimación de la eficiencia institucional: El caso de las dependencias de educación superior (DES) de ingeniería de México Measuring the institutional efficiency using data envelopment analysis and artificial neural networks. Revista Electronica Nova Scientia Vol. 6 (2), 356-378.

Andersen, P., y Petersen, N. (1993). Aprocedere for ranking efficient units in Data Envelopment Analysis. Mngt Sci 39(10), 1261-1264.

Aristovnik, A., y Obadic, A. (2014). Measuring relative efficiency of secundary education in selected EU and OECD countries: The cese of Slovenia and Croatia. Technological and Economic Development of Economy 20(3), 419-451.

Balaguer, M., Prior, D., y Tortosa, A. (2007). On the determinants of local goverment performance: A two-stage nonparametric approach. European Economic Review 51(2), 425-451.

Banker, R., y Natajaran, R. (2008). Evaluating contextual variables affecting productivity using Data Envelopment Analysis. Operation Research 17 (1), 48-58. 
Barney, J. (1991). Firm resources and sustained competitive advantage. Journal of Management, 99-120.

Bass, B., y Avolio, B. (1997). Full Range Leadership Development: Manual for the Multifactor Leadership Questionnaire. Mind Garden.

Battese, G., y Rao, D. (2002). Technology gap, efficiency, and a stochastic metafrontier function. Int J Bus 1(2), 87-93.

Battese, G., Rao, D., y O'Donnell, C. (2004). A metafrontier production function for estimation of technical efficiencies and technology gaps for firms operating under different technologies. Prod Anal 21(1), 91-103.

Borghans, L., y Schils, T. (2012). The leaning tower of PISA: The effect of test motivation on scores in the international student assessment. The Society of Labor Economists.

Bradley, S., Johnes, G., y Millington, J. (2001). The effect of competition on the efficiency of secondary schools in England. European Journal of Operational Research 135(3), 545-568.

Bray, M., y Thomas, R. (1995). Levels of comparison in educational studies: Different insights from different literatures and the value of multilevel analyses. Harvard Educational review 65(3), 472-490.

Brennan, S., Haelermans, C., y Ruggiero, J. (2014). Nonparametric estimation of education productivity incorporating nondiscretionary inputs with an application to Dutch schools. European Journal of Operational Research 234(3), 809-818.

Brunello, G., y Checchi, D. (2007). Does school tracking affect equality of opportunity? New international evidence. Economic Policy 22(52), 781-861.

Cazáls, C., Florens, J., y Simar, L. (2002). Nonparametric frontier estimation: A robust approach. Journal Econometrics 106(1), 1-25.

Cherchye, L., De Witte, K., Ooghe, E., y Nicaise, I. (2010). Efficiency and equity in private and public education: A nonparametric comparison. European Journal of Operational Research 202(2), 563-573.

Coco, G., y Lagravinese, R. (2014). Cronyism and education performance. Economic Modelling 38, $443-450$.

Coelli, T., Rao, P., O’Donnell, C., y Battese, G. (2005). An Introduction to Efficiency and Productivity Analysis. New York: Springer.

Cooper, W., Seiford, L., y Tone, K. (2000). Data Envelopment Analysis: A Comprehensive Text with Models, Applications, References and DEA-Solver Software. Boston: Kluwer Academic Publishing.

Cordero, J., Pedraja, F., y Santín, D. (2010). Enhancing the inclusion of non-discretionary inputs in DEA. Journal of Operational Research Society 61(4), 574-584.

Cordero, J., Santín, D., y Simancas, R. (24 de june de 2017). Assessing European primary school performance through a conditional nonparametric model. Journal of the Operational Research Society 68, 364-376. Obtenido de Journal of the Operational Research Society advance online publication: doi:10.1057/jors.2015.42

Crespo, E., Pedraja, F., y Santín, D. (2014). Does school ownership matter? An unbiased efficiency comparison for regions of Spain. Journal of Productivity Analysis 41(1), 153-172. 
Cuervo, A. (1993). El papel de la empresa en la competitividad. Papeles de Economía Española 56, 363-378.

Daraio, C., y Simar, L. (2005). Introducing environmental variables in nonparametric frontier models: A probabilistic approach. Journal of Productivity Analysis 24(1), 93-121.

Daraio, C., y Simar, L. (2007). Advanced robust and nonparametric methods in efficiency analysis. Methodology and Applications. New York: Springer.

De Jorge, J., y Santín, D. (2010). Determinantes de la eficiencia educativa en la Unión Europea. Hacienda Publica Española 193(2), 131-155.

De Witte, K., y Kortelainen, M. (2013). What explains performance of students in a heterogeneous environment? Conditional efficiency estimation with continuous and discrete environmental variables. Applied Economics 45(17), 2401-2412.

De Witte, K., y López, L. (2015). Efficiency in education. A review of literature and a way forward. Journal of the Operational Research Society.

De Witte, K., y Marques, R. (2009). Capturing the environment, a metafrontier approach to the drinking water sector. International Transactions of Operational Research 16(2), 257-271.

De Witte, K., y Rogge, N. (2011). Accounting for exogenous influences in performance evaluations of teachers. Economics of Education Review 30(4), 641-653.

De Witte, K., Thanassoulis, E., Simpson, G., Battisti, G., y M, C. (2010). Assessing pupil and school performance by nonparametric and parametric techniques. Journal of the Operational Research Society 61(8), 1224-1237.

Deprins, D., Simar, L., y Tulkens, H. (2006). Measuring labor efficiency in post offices. Boston: Springer.

Deutsch, J., Dumas, A., y Silber, J. (2013). Estimating an educational production function for five countries of Latin America on the basis of the PISA data. Economics of Education Review $36,245-262$.

Dronkers, J., y Robert, P. (2008). Differences in scholastic achievement of public, private governmentdependent, and private independent schools a cross-national analysis. Educational Policy 22(4), 541-577.

Dufrechou, P. A. (2016). The efficiency of public education spending in Latin America: A. International Journal of Educational Development, 188-203.

Essid, H., Ouellette, P., y Vigeant, S. (2014). Productivity, efficiency, and technical change of Tunisian schools: A bootstrapped Malmquist approach with quasi-fixed inputs. Omega 42(1), 88-97.

Farrell, M. (1957). The measurement of productive efficiency. Journal of the Royal Statistical Society $120,253-281$.

Farrell, M., y Fieldhouse, M. (1962). Estimating efficient production functions under increasing returns to scale. Journal of the Royal Statistical Society 125, 252-267.

Giambona, F., Vassallo, E., y Vassiliadis, E. (2011). Educational systems efficiency in European Union countries. Studies in Educational Evaluation 37(2), 108-122.

Goldstein, H. (2003). Multilevel Statistical Models. London: Arnold Publisher. 
Haelermans, C., y De Witte, K. (2012). The role of innovations in secondary school performance: Evidence from a conditional efficiency model. European Journal of Operational Research 223(2), 541-549.

Hanushek, E. (1979). Conceptual and empirical issues in the estimation of educational production functions. Journal of Human Resources 14(3), 351-388.

Hanushek, E., y Woessman, L. (2011). The economics of international differences in educational achievement. En E. Hanushek, S. Machin, y L. Woessmann, Handbook of the Economics of Education (págs. 89-200). Amsterdam.

Heckman, J. (2011). Integrating personality psychology into economics. National Bureau of Economic Research.

Herve, L. (2006). A linear programming framework for free disposal hull technologies and cost functions: Primal and dual models. European Journal of Operational Research $168,340-344$.

Jerrim, J. (2015). Why do East Asian children perform so well in PISA? An investigation of Westernborn children of East Asian descent. Oxford Review of Education Vol. 41.

Johnes, J. (2006). Measuring efficiency: A comparison of multilevel modelling and data envelopment analysis in the context of higher education. B Econ Res 58(2), 75-104.

Koopmans, T. (1951). Analysis of production as an efficient combination of activities. En T. Koopmans, Activity analysis of production and allocation (págs. 33-97). New York: Wiley.

Kramer, G. (1983). The ecological fallacy revisited: Aggregate versus individual level-findings on economics and elections, and sociotropic voting. Am Polit Sci Rev 77(1), 92-111.

Lavy, V. (2015). Do differences in school's instruction time explain international achievement gaps in Maths, Science and Language? Evidence from developed and developing countries. The Economic Journal , 397-424.

O’Donnell, C., Rao, D., y Battese, G. (2007). Metafrontier frameworks for the study of firm-level efficiencies and technology ratios. Empirical Econ 34(2), 231-255.

Portela, M., y Thanassoulis, E. (2001). Decomposing school and school-type efficiency. Eur J Opl Res 132(2), 357-373.

Racine, J., Hart, J., y Li, Q. (2006). Testing the significance of categorical predictor variables in nonparametric regression models. Econometric Reviews 25(4), 523-544.

Rivkin, S., y JC, S. (2015). Instruction Time, Classroom Quality, and Academic Achievement. The Economic Journal, vol 125(588), 425-448.

Rowe, K. (2000). Assessment, League Tables and School Effectiveness: Consider the Issues and 'Let's Get Real'. Journal of Educational Enquiry, Vol. 1(1), 73-98.

Ruggiero, J. (1996). On the measurement of technical efficiency in the public sector. European Journal of Operational Research 90(3), 553-565.

Ruggiero, J. (2000). Nonparametric estimation of returns to scale in the public sector with an application to the provision of educational services. Journal of the Operational Research Society 51(8), 906-912. 
Sammons, P., Nuttall, D., y Cuttance, P. (1993). Differential school effectiveness: Results from a reanalysis of the Inner London Education Authority's junior school project data. Brit Educ Res J 19, 381-405.

Sammons, P., Thomass, E., Motimore, P., Owen, C., y Pennell, H. (1996). Assessing School Effectiveness: Developing Measures to Put School Performance in Context. Manchester: OFSTED, Publications Centre.

Schmidt, P. (1976). On the statistical estimation of parametric frontier production functions. The Review of Economics and Statistics (58), 238-289.

Schuetz, G., y HL, U. (2008). Education policy and equality of opportunity. Kyklos 61(2, 279-308.

Simar, L., y Wilson, P. (2007). Estimation and inference in two-stage, semi-parametric models of productive processes. Journalof Econometrics, 136 (1), 31-64.

Simar, L., y Wilson, P. (2011). Two-stage DEA: caveat emptor. Journal of Productivity Analysis, 205-218.

Spiezia, V. (2010). Does computer use increase educational achievements? Student-level evidence from PISA. Economic Studies 1, 1-22.

Täht, K., y Must, O. (2013). Comparability of educational achievement and learning attitudes across nations. Educational Research and Evaluation 19(1), 19-38.

Thanassoulis, E. (1999). Setting achievement targets for school children. Educ Econ 7(2), 101-119.

Thanassoulis, E., y Portela, M. (2002). School outcomes: Sharing the responsibility between pupil and school. Educ Econ 10(2), 183-207.

Thieme, C., Giménez, V., y Prior, D. (2012). A comparative analysis of the efficiency of national education systems. Asia Pacific Education Review 13(1), 1-15.

Thieme, C., Prior, D., y Tortosa, A. (2011). Desempeño de los centros educativos: ¿un problema de recursos o capacidades organizativas? Revista de Economía Pública 199-(4/2011), 81-118.

Thieme, C., Prior, D., y Tortosa, A. (2013). A multilevel decomposition of school performance using robust nonparametric frontier techniques. Economics of Education Review 32, 104-121.

Urwick, J., y Junaidu, S. (1991). The effects of school physical facilities onthe processes of education: A qualitative study of Nigerian primary schools. International Journal of Educational Development 11(1), 19-29.

Vandenberghe, V., y Robin, S. (2004). Evaluating the effectiveness of private education across countries: A comparison of methods. Labour Economics 11(4), 87-506.

Worthington, A. (2001). An empirical survey of frontier efficiency measurement techniques in education. Education Economics 9(3), 245-268.

Zhang, Y., y Bartels, R. (1998). The effect of sample size on the mean efficiency in DEA with an application to electricity distribution in Australia. J Prod Anal 9(3), 187-204.

Zhao, Y., Zhang, G., Yang, W., Kirkland, D., Han, X., y Zhang, J. (2008). A comparative study of educational research in China and the United States. Asia Pacific Journal of Education 28(1), 1-17. 